\title{
The Suitability of BV2 Cells as Alternative Model System for Primary Microglia Cultures or for Animal Experiments Examining Brain Inflammation
}

\author{
Anja Henn ${ }^{1}$, Søren Lund ${ }^{2}$, Maj Hedtjärn ${ }^{3}$, André Schrattenholz ${ }^{4}$, Peter Pörzgen ${ }^{5}$ and Marcel Leist ${ }^{1}$ \\ ${ }^{1}$ Doerenkamp-Zbinden Chair for alternative in vitro methods to replace animal experiments, University of Konstanz, Konstanz, \\ Germany; ${ }^{2}$ Novo Nordisk A/S, Bagsværd, Denmark; ${ }^{3}$ Santaris Pharma A/S, Hørsholm, Denmark; ${ }^{4}$ ProteoSys AG, Mainz, \\ Germany; ${ }^{5}$ Hawaii Pacific University, Kaneohe, USA
}

\section{Summary}

The role of microglia in neurodegeneration, toxicology and immunity is an expanding area of biomedical research requiring large numbers of animals. Use of a microglia-like cell line would accelerate many research programmes and reduce the necessity of continuous cell preparations and animal experimentation, provided that the cell line reproduces the in vivo situation or primary microglia (PM) with high fidelity. The immortalised murine microglial cell line BV-2 has been used frequently as a substitute for PM, but recently doubts were raised as to their suitability. Here, we re-evaluated strengths and potential shortcomings of $B V-2$ cells. Their response to lipopolysaccharide was compared with the response of microglia in vitro and in vivo. Transcriptome (480 genes) and proteome analyses after stimulation with lipopolysaccharide indicated a reaction pattern of $B V-2$ with many similarities to that of $P M$, although the average upregulation of genes was less pronounced. The cells showed a normal regulation of $N O$ production and a functional response to IFN- $\gamma$, important parameters for appropriate interaction with $T$ cells and neurons. BV-2 were also able to stimulate other glial cells. They triggered the translocation of $N F-\varkappa B$, and a subsequent production of IL-6 in astrocytes. Thus, BV-2 cells appear to be a valid substitute for PM in many experimental settings, including complex cell-cell interaction studies.

Keywords: microglia, inflammation, $B V-2$, transcriptome, replacement

\section{Introduction}

Microglia are the resident macrophage-like cells of the central nervous system (CNS) with a broad role in the brain's innate immunity and in inflammatory neuropathologies (Nelson et al., 2002). They have been examined in hundreds of studies in animal models and in primary cultures. As their proliferation capacity is limited, they have to be isolated freshly for each experiment. For a typical preparation of rodent microglia, 15-30 brains are required to yield cells for a limited amount of experiments on signalling or disease mechanisms. For biochemical work or chip expression analysis, considerably higher numbers are required. This has a large impact on overall animal consumption in biomedical research. A cell line alternative would be highly desirable, as it would save animals, time and valuable consumables, and facilitate research work.

Microglia show great functional plasticity when activated. They are equipped with a broad range of pattern-recognition receptors of the toll-like receptor family (TLR family) to detect microbial intruders and brain damage (Lee and Lee, 2002; Falsig et al., 2008). Most work on microglial activation and signalling has been performed in vitro, frequently by using cell lines such as N9 (Corradin et al., 1993) and BV-2 (Blasi et al., 1990). Data on primary microglia (PM), isolated from brain cultures of neonatal pups (Giulian and Baker, 1986) or directly from adult mice (Baker et al., 2002) are more restricted, due to the limited yield of biological material. In addition, some ex vivo studies have been performed with freshly isolated microglia from diseased brains (Baker et al., 2002).

$\mathrm{BV}-2$ cells were derived from raf/myc-immortalised murine neonatal microglia and are the most frequently used substitute for primary microglia. They have been used e.g. for pharmacological studies (Lund et al., 2005), studies of phagocytosis (Hirt and Leist, 2003) and for many important immunological discoveries in altogether at least 200 publications. With respect to neurodegeneration studies, it is important that BV-2, similar 
to primary microglia, express functional NADPH oxidase, an enzyme frequently implicated in microglia-triggered neuronal damage (Wu et al., 2006; Yang et al., 2007). However, doubts have been raised that this cell line does not always model the reaction of primary microglia in culture or in the brain (Hausler et al., 2002; de Jong et al., 2008; Horvath et al., 2008). In one study, BV-2 were compared to primary rat microglia, introducing a bias of species differences and different analysis methodology, e.g. for cytokine ELISAs. In another approach, data were obtained on the comparison of primary murine microglia and in vivo microglia activation. In this study, some proteins induced in $\mathrm{BV}-2$ were shown to correspond to upregulated genes in primary microglia (Lund et al., 2006).

In extension of this study, we sought here to broadly characterise the BV-2 inflammatory response in comparison to primary microglia and microglia in vivo. Most inflammatory mediators are regulated on the transcriptional level, and gene expression profiling allows the examination of multiple endpoints simultaneously. As a consequence, there are great expectations that this approach may help to characterise the usefulness and limitations of BV-2 as an alternative in vitro model, without resorting only to some randomly chosen endpoints and assays. We present here the lipopolysaccharid (LPS) response pattern of microglia and BV-2. We focused on about 500 inflammation-related genes analysed by competitive hybridisation. Finally, the outcome of these studies was correlated with data from proteomics analysis, with in vivo microglia analysis, and with functional capacities of BV-2 cells relevant for various biological questions.

\section{Animals, materials and methods}

\subsection{Materials and chemicals}

Tissue culture material was obtained from Greiner Bio-One GmbH (Frickenhausen, Germany), media, phosphate-buffered saline (PBS), antibiotics and foetal bovine serum (FBS) were obtained from GIBCO (Invitrogen, Karlsruhe, Germany) and LPS (Salmonella abortus equi) was purchased from BioCloth (Aidenbach, Germany).

\subsection{Animals and in vivo experimentation}

All experimental procedures were carried out in accordance with national (directive of the Danish National Committee on Animal Ethics) and international laws and policies (EEC Council Directive 86/609, OJ L 358, 1, Dec.12, 1987; Guide for the Care and Use of Laboratory Animals, U.S. National Research Council, 1996). Pregnant C57bL/6J and male C57bL/6J mice (3 months of age) were purchased from M \& B (Lille Skensved, Denmark). For the current study, no new animal experiments were performed; instead historic animal data were used for comparison. Mice were treated as described in detail by Lund et al. (Lund et al., 2006).

\subsection{Primary cultures}

Primary microglia cultures were prepared as initially described by Giulian and Baker (Giulian and Baker, 1986) using the adaptations described earlier (Lund et al., 2005; 2006). Primary cor- tical astrocytes were prepared according to a slightly modified version of a protocol by David E. Weinstein (Weinstein, 1997) as described in detail earlier (Falsig et al., 2004; 2006)

\subsection{Standard cell incubation scheme for array (PM and BV-2) and proteomics experiments (BV-2)}

All cell incubations were performed at $37^{\circ} \mathrm{C}, 5 \% \mathrm{CO}_{2}$ and $95 \%$ relative humidity. Suspended PM (see above) were seeded at 3 million cells/Petri dish (surface area $20 \mathrm{~cm}^{3}$ ) in $5 \mathrm{ml} \mathrm{me-}$ dium. After $25 \mathrm{~min}$ of incubation, loosely adherent cells were removed by tapping the sides of the dish, followed by two washes in PBS. After overnight incubation, cells were washed once in PBS, followed by addition of $5 \mathrm{ml}$ medium (1\% FBS). Cells were stimulated with LPS $(100 \mathrm{ng} / \mathrm{ml})$ for 4 or $16 \mathrm{~h}$, and $100 \mu 1$ of the supernatant was sampled for cytokine analysis before cells were harvested for RNA extraction.

BV-2 cells (murine microglia, kindly provided by E. Blasi, Perugia) (Blasi et al., 1990) were maintained in Roswell Park Memorial Institute (RPMI) medium 1640 supplemented with $10 \% \mathrm{FBS}$ and antibiotics (penicillin $100 \mathrm{U} / \mathrm{ml}$, streptomycin $100 \mu \mathrm{g} / \mathrm{ml}$ ). Antibiotics were omitted for the functional studies and also for maintenance during the later parts of the study. Stimulations of BV-2 were always performed in $2 \%$ FBS. BV-2 cells were cultured as described for PM, except that only half the number of cells was plated and RPMI was replaced by Dulbecco's Modified Eagle Medium (DMEM). For proteomics analysis the BV-2 cells were stimulated for $24 \mathrm{~h}$, washed twice with PBS, and then lysed in $2 \%$ SDS with $0.1 \mathrm{M}$ Tris $(\mathrm{pH}$ 8.8). In all experiments viability of the cells was controlled by various standard methods as described earlier (Leist et al., 1997; Volbracht et al., 1999; Latta et al., 2000).

\subsection{Cytokine and nitrite determination}

The murine cytokines interleukin-6 (IL-6) and tumour necrosis factor- $\alpha$ (TNF- $\alpha$ ) were measured in MaxiSorp plates from Nunc (Langenselbold, Germany) using murine specific OptEIA $^{\text {TM }}$ ELISA kits from Pharmingen (Brøndby, Denmark) according to the manufacturer's protocol.

Nitrite [surrogate marker for nitric oxide (NO)] was measured by use of the Griess reagent from Sigma-Aldrich. In brief, $70 \mu 1$ supernatant or $\mathrm{NaNO}_{2}$ standards were mixed with $30 \mu 1 \mathrm{~N}-(1$ naphtyl) ethylendiamine $\left(0.1 \%\right.$ in $\left.\mathrm{H}_{2} \mathrm{O}\right)$ and $30 \mu 1$ sulfanilamide $(1 \%$ in $1.2 \mathrm{~N} \mathrm{HCl})$ in a 96-well plate. After $3 \mathrm{~min}$, samples were read at $(570-690 \mathrm{~nm})$ in a spectrophotometer.

\subsection{NF- $\kappa$ B translocation}

For quantification of nuclear factor $\kappa \mathrm{B}(\mathrm{NF}-\kappa \mathrm{B})$ translocation, cells were plated at 10,000 cells/well in DMEM with 10\% FBS. After one week of incubation, the FCS concentration was reduced to $2 \%$ FCS. The cells were treated for one hour with BV2-conditioned medium (CM) or LPS-control and then fixed with $4 \%$ paraformaldehyde for 10 min. After permeabilisation with $0.1 \%$ Triton-X100 in PBS, the cells were blocked with $10 \%$ FCS in PBS. The primary antibody (purified mouse anti-NF- $\kappa B$ p65, clone: $20 / \mathrm{NF}-\kappa \mathrm{B} / \mathrm{p} 65$, final dilution: 1:200) was purchased from BD Biosciences (San Jose, CA USA), and the binding was visualised with an Alexa-488-labelled secondary antibody (Sig- 
ma-Aldrich, St. Louis, MO, USA). Cells were counterstained with $\mathrm{H}-33342$ and the nuclear translocation/intensity of NF-кB was quantified with a Cellomics ArrayScan ${ }^{\mathrm{TM}}$, using the predefined algorithm "molecular translocation". The principle is based on cell image acquisition with a high resolution CCD camera, followed by automatic identification of cells (based on nuclear staining with $\mathrm{H}-33342$ ). The nuclear-cytoplasmic ratio of antigen (NF-кB p65) signal intensity was quantified by dividing the average antigen intensity in the nuclear area (A) by the average antigen intensity of a ring around this area, that covered a cytoplasmic rim (B). "Activated cells" were defined as the percentage of cells whose $\mathrm{A} / \mathrm{B}$ ratio was one standard deviation above the average $\mathrm{A} / \mathrm{B}$ ratio obtained automatically from the reference wells containing untreated control cells.

\subsection{BV-2 conditioned medium (CM)}

BV-2 were seeded in a T175 flask and incubated overnight in DMEM and $2 \%$ FCS. Cells were treated with $50 \mathrm{ng} / \mathrm{ml}$ LPS in $25 \mathrm{ml} \mathrm{DMEM}$ and $2 \%$ FCS for various time points. After the incubation, the supernatant (=CM) was filtered with a 0.22 $\mu \mathrm{m}$ sterile filter $\left(\mathrm{TPP}^{\circledR}\right.$; Trasadingen, Switzerland) to remove cells and cell debris. For LPS-control medium, BV-2 were incubated for $24 \mathrm{~h}$ with medium only. LPS $(50 \mathrm{ng} / \mathrm{ml})$ was then added to filtered medium afterwards. Aliquots were frozen in liquid nitrogen and stored at $-80^{\circ} \mathrm{C}$. For activation of primary astrocytes, CM was thawed and pre-warmed. Astrocyte growth medium was then removed and substituted with different variants of CM.

\subsection{RNA extraction, RT PCR and quantitative PCR}

Total RNA was extracted using TRIzol from Invitrogen and 1 $\mu \mathrm{g}$ of total RNA was reverse transcribed with Superscript ${ }^{\mathrm{TM}}$ II Reverse Transcriptase (Invitrogen) using random hexamers and OligodT-Primer in a $20 \mu 1$ reaction according to the manufacturer's protocol. PCR amplification of the cDNA was quantified using the Platinum ${ }^{\circledR}$ SYBR ${ }^{\circledR}$ Green qPCR SuperMix-UDG kit (Invitrogen). Using the iCycler data analysis software (Bio-Rad, Hercules, CA, USA), the threshold cycle $\left(\mathrm{C}_{\mathrm{T}}\right)$ was determined for each sample. The amplified cDNA levels were compared among different groups using the delta-delta method. Primers used were gapdh sense (NM_008084): 5'-TGC ACC ACC AAC TGC TTA G-3', anti-sense: 5'-GGA TGC AGG GAT GAT GTT C-3’ ${ }^{\prime}$ ill $-b$ sense (NM 008361.3): 5' - TTT TTG TTG TTC ATC TCG GAG CCT GTA G-3’, anti-sense: 5'- GAG CAC CTT CTT TTC CTT CAT CTT TG-3'. Ccl2 sense (NM_011333): 5'-CAT GCT TCT GGG CCT GCT GTT C-3', anti-sense 5'CCT GCT GCT GGT GAT CCT CTT GTA G-3’. rigl sense (NM_172689): 5'- GAC CAG AGC ACT CGT GGA TG -3', anti sense: 5'- GGA GCG TCA TTC CTG TTG CC-3’' ifit3 sense (NM_010501): 5'- GGC TGA ATG GTT TTG GGG TTT G-3', anti sense: 5'- CAG GTT CAT GGT GCC AGG AAA A-3'. $u-p a$ sense (NM_008873.2): 5'- AGG TTT ACT GAT GCT CCG TTT GGT TC-3', anti-sense: 5'- TTT ACG ACG GAC ATT TTC AGG TTC TTT-3'. $t d k-i$ sense (NM_020557): 5'- GGA TGC GCT GCA CAC CAA TT -3', anti-sense 5'- TAG CTC CTT GGC CTC CGT TTG -3’ $i$-nos sense (NM_010927): 5'- TTG CCA CGG ACG AGA CGG ATA GG -3', anti-sense
5'- GGG CAC ATG CAA GGA AGG GAA CTC -3'. tlr2 sense (NM_011905.2): 5'- CCT CCG TCT TGG AAT GTC ACC AGG -3', anti-sense 5'- GAG CCA CGC CCA CAT CAT TC $-3^{\prime}$. $t$ tr3 sense (NM_126166.3): 5'- GCC CCC TTT GAA CTC CTC TTC -3', anti-sense 5'- AGA TCC TCC AGC CCT CGA TG -3'. tlr4 sense (NM_021297.2): 5'- GAG TCA GAA TGA GGA CTG GGT GAG -3', anti-sense 5'- GGA ATA AAG TCT CTG TAG TGA AGG CAG -3'. gsrl sense (NM_010344): 5'AGA TGT TGA CTG CCT GCT CTG G -3', anti-sense 5' - TCT CCC ACA GCA TAG ACG CC -3'. nod2 sense (NM_145857): 5'- CAG GCA GCT CAG GTT GAC TCT G -3', anti-sense 5'TAG AAA GCG GCA AAA AAG CAC TGG AAG -3’.

\subsection{Transcript analysis by oligonucleotide hybridisation analysis (Neuroflame)}

A list of mouse cytokines, apoptotic mediators and inflammatory factors was compiled (Falsig et al., 2006; Lund et al., 2006). For each of these genes one oligonucleotide (40-50mer) was designed by MWG (MWG, Ebersberg, Germany) using their proprietary Oligo4array software and CodeSeq database, which selects the oligos preferentially from the 3'-region of each coding sequence. The oligomers were spotted onto activated glass slides (Pan Epoxy, MWG or CodeLink, Amersham) using a 417 Affymetrix (ring and pin) spotter. Sample preparation and labelling was carried out as described (Lund et al., 2006). Total RNA (12-15 $\mu \mathrm{g}$ ) was reverse transcribed using random hexamers, incorporating amino-allyl dUTP into the $1^{\text {st }}$ strand cDNA. Cy-3 or Cy-5 dye esters, respectively were coupled to the cDNA samples. Each slide was scanned in a 428 Affymetrix confocal laser scanner at three different intensities (photo multiplier gains). $\mathrm{Cy} 3 / \mathrm{Cy} 5$ ratio normalisation was carried out by multiplying each ratio value with a scaling factor, which was defined as the ratio of the overall signal intensity of the Cy5 versus $\mathrm{Cy} 3$ channel (Knudsen, 2002). Each microarray experiment was performed at least twice independently, each with duplicates. To further account for bias introduced by dye bleaching or labelling, each experiment was carried out as dye-swap experiment with the resulting ratio value being the arithmetic mean from two slides of oppositely labelled sample pairs.

\subsection{Array statistics}

For each time point, two independent biological experiments were performed. For each data point, total RNA was extracted from six animals and pooled before hybridisation. Each pool of RNA was split, and then one fraction each was labelled with Cy3 and Cy5. Competitive hypridizations on the chip were performed in both directions (dye swap). Thus, a total of four hybridisations (chip experiments) was obtained for each time point analyzed. Genes regulated $\geq 1.8$ fold (up or down) in 3 out of 4 hybridisations were considered regulated, but only if they had a signal intensity of more than 5 fold above background. Equivalent numbers of hybridisations and threshold values were used to identify gene regulations in BV-2 and primary microglia cells. The material for a competitive hybridisation (LPS $v s$. control) was however pooled from 8 independent experiments per condition. Neuroflame values displayed in Table 1 are the arithmetic means of the 4 ratios pertaining to a given experiment. 


\subsection{Proteomics analysis}

The differential and quantitative protein expression analysis was performed as described previously (Groebe et al., 2007) and is based on radio-iodination, 2D-PAGE and high sensitivity radio imaging. In brief, small amounts of each sample were labelled with ${ }^{125} \mathrm{I}$ and ${ }^{131} \mathrm{I}$ for differential pattern control. The signals from these two isotopes were used for statistical treatment of abundance differences (Schrattenholz and Groebe, 2007). Spots were analysed first with a high throughput peptide mass fingerprint procedure based on MALDI-TOF-MS. For those spots for which no unambiguous identification was achieved a fragment ion analysis based on LC-ESI-IonTrap-MS/MS was added (Lund et al., 2006; Groebe et al., 2007).

\section{Results}

\subsection{The inflammatory gene pattern trigged by LPS in BV-2 cells}

BV-2 showed a broad response of gene activation after exposure to LPS with many different types of genes activated (Tab. 1). We used primary microglia (PM) data published earlier (Lund et al., 2006) for a comparison of the transcriptional responses of PM and BV-2. The experiments and analyses were performed for all cell types in exactly the same way. This comparison showed that BV-2 cells have an overall response pattern that parallels that of PM. Virtually all (90\%) of those genes that were regulated in BV-2 were also found in PM. However,

Tab. 1: Upregulation of genes by LPS in primary microglia and BV-2 cells

Primary murine microglia (PM) or BV-2 cells were stimulated with LPS (100 ng/ml) for 4 or $16 \mathrm{~h}$ before isolation of mRNA. Transcriptional changes were examined by chip analysis using Neuroflame arrays. Genes up-regulated significantly in BV-2 were selected for display of their regulation (numbers = fold upregulation) in PM and BV-2. Only statistically significant data are displayed. The transcripts that were significantly increased in PM, but not BV-2 are listed below. Their gene identifier and the extent of up-regulation, as well as the genes that were present on the chip, but not regulated at all may be retrieved from (Lund et al., 2006): BID, BID3, Bcl2-like 11, Birc1e, Birc2, Birc3, CFLAR, CHOP-10, Daxx, Fas, TNFrsf5, Cox-1, Cox-2, Phospholipase a2, PIK3C2gamma, Sphingosine kinase1, u-Pa, VCAM1, AGTRlike1, BACE, Bcl3, HspA5, Cathepsin m, Cd83 antigen, Clic4, Coagulation factor III, Cytochrome P450 IV, Disc1, MyD118, GRK4, GRK6, IER3, NaC1, Nurr77, Peroxiredoxin 5, PHLDA1, Pleiotropin, PNP, RELB, Trim30, Trem3, Ubiquitin-protein-ligase, VMAT2, BALB/c gp49b gene, casp1, Cd86 antigen cd86, CCl2, CCl7, CXCL5, RDC1, CCR1, CCRL2, CCR2, CSF2, Endothelin 1, IL12Rb2, IL-1ra, IL23a, IL18, IRAK3, NGFb, OSM, OSM-R, C3, CAT2, GBP, IFNB, Irg1, iNOS, MSR1, Myeloperoxidase, NFKB-P49/p100, SOD1, SOD2, TLR1, H-2I gene for class $1 \mathrm{MHC}$ glycoprotein, pBR, Proteasome SU28-beta, PSMB9

Cytokines / Chemokines

Chemokine ligand 3, MIP-1 alpha, Ccl3

Chemokine ligand 4, MIP-1 beta, Ccl4

Interleukin 1 beta

\begin{tabular}{|c|c|c|c|}
\hline \multicolumn{2}{|c|}{ BV-2 } & \multicolumn{2}{c|}{ PM } \\
\hline $\mathbf{4 h}$ & $\mathbf{1 6 h}$ & $\mathbf{4 h}$ & $\mathbf{1 6 h}$ \\
\hline 6.6 & 2.6 & 23.3 & 10.3 \\
\hline 6.5 & 3.6 & 27.6 & 4.1 \\
\hline 13.5 & & 141.9 & 10.8 \\
\hline
\end{tabular}

Immune receptors and associated genes

\begin{tabular}{|l|c|c|c|}
\hline Inter-Cellular Adhesion Molecule 1, ICAM1 & 4.0 & 4.1 \\
\hline I(Kappa)B(alpha), NFkBia & 4.7 & 4.8 & 4.8 \\
\hline NFkB1-P50/105 & 2.3 & 3.6 & 2.4 \\
\hline Toll-like receptor 2,TLR 2 & 6.2 & 2.8 & 7.0 \\
\hline Glycoprotein 49a, gp49a & 2.7 & 2.2 \\
\hline Cd209a antigen & 2.5 & 2.2 & 3.9 \\
\hline Suppres s or of cytokine signaling 3,SOCS3 & 1.9 & 28.6 & 19.9 \\
\hline
\end{tabular}

Cell death

\begin{tabular}{|c|c|c|c|c|}
\hline B-cell leukemia 2 related protein a1a, BclA1a & 4.5 & & 10.0 & 4.5 \\
\hline Caspase9 & 5.2 & 2.5 & 16.3 & 5.6 \\
\hline Caspase4/caspase11 & 2.9 & 3.6 & 8.0 & 4.3 \\
\hline
\end{tabular}

Stress related genes

\begin{tabular}{|c|c|c|c|c|}
\hline Ccaat/enhancer binding protein - delta & 5.0 & 2.4 & 2.5 & 2.9 \\
\hline Thymidylate kinase, Ips-inducible member, TDKi & & 2.0 & 45.2 & 3.4 \\
\hline Interferon-ind. prot. with tetratricopeptide rep. 1, Ifit1 & 2.0 & & 23.2 & 6.9 \\
\hline Interferon-ind. prot. with tetratricopeptide rep. 3 ,Ifit3 & & 9.7 & 25.2 & 3.6 \\
\hline Interferon regulatory factor 1 , IIf1 & 2.0 & & 6.8 & \\
\hline Serum amyloid a2, SAA2 & 14.9 & & 4.1 & \\
\hline Serum amyloid a3, SAA3 & 4.5 & & 3.7 & \\
\hline
\end{tabular}


the $\mathrm{BV}-2$ response was weaker and narrower than the response of PM. Only $17 \%$ of the genes detected to be significantly regulated in PM were also detected in this analysis in BV-2 (Fig. 1A). One theoretical explanation for the limited number of gene inductions may be a high basal activation state of BV-2 cells. However, the levels of inflammatory mediators (TNF- $\alpha$, IL-1 $\beta$ and NO) in non-stimulated cultures were always under the detection limit of conventional detection methods, and were greatly enhanced upon LPS stimulation. On that basis, a high basal activation state of BV-2 cells was ruled out. This is also in agreement with data from earlier work, where we found solid activation of inflammation related kinases (JNK and p-38) as well as transcription factors (c-JUN and NF- $\mathrm{BB}$ ) when resting cells (primary or BV-2) were stimulated with LPS (Lund et al., 2005). Another explanation could be that the rich inflammatory profile observed for primary microglia results from transcripts originating from contaminating cells in the cultures. However, microglial cultures are routinely established in our and other labs with high purity. We characterised our cultures extensively with biochemical and immunocytochemical methods, and contaminations with neurons, astrocytes or endothelial cells were
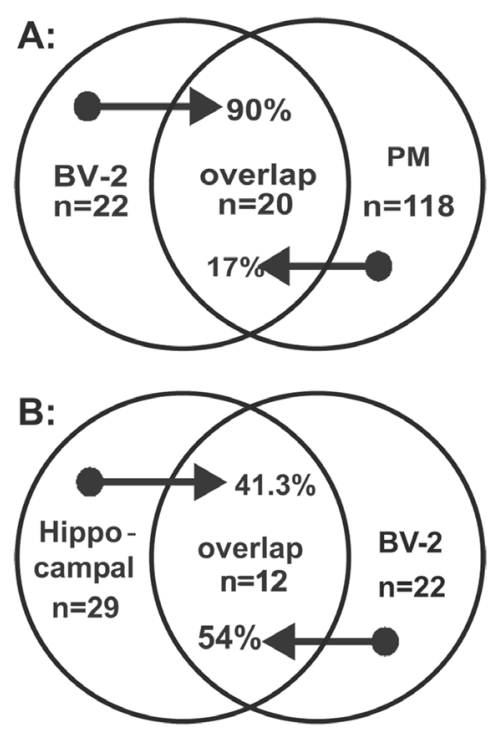

Fig. 1: Overlap of genes regulated by LPS in PM and in BV-2 cells

Mice (i.c.v. injection), primary microglia (PM) or BV-2 cells were stimulated with LPS. (A) The overlap is indicated in percent of regulated genes on the Neuroflame chip when comparing BV-2 to $\mathrm{PM}$ or vice versa. (B) Comparison of hippocampal microglial gene regulations (in vivo) with BV-2. Genes were considered regulated $(n)$, when they exceeded $\geq 1.8$ fold average induction in their respective systems at least at one time point. The percentage numbers with right-pointing arrow indicate how many percent of the genes to the left are also regulated in the system on the right. The percentage numbers with left-pointing arrow indicate how many percent of the genes to the right are also regulated in the system on the left. below $5 \%$. Furthermore, the broad transcriptional pattern identified here is relatively similar to that of LPS-stimulated macrophages (Rosenberger et al., 2000). Microglia are believed to adopt many features of macrophages when stimulated (Kappler et al., 1997; Qin et al., 2005), which makes this overlap in response pattern very plausible.

\subsection{In vivo transcriptional response of microglia compared to the response of LPS-stimulated BV-2}

The most relevant comparison for the fidelity of the BV-2 system is not the neonatal PM culture, but the real reaction of microglia in vivo. We made use of a unique set of well-characterised data on in vivo activation of microglia (Lund et al., 2006) for this comparison. I.c.v. injection of LPS induced a robust expression of several inflammatory gene clusters also identified in vitro. Notably, the temporal dynamics of the cerebral inflammation was distinct from that observed in vitro. The response peaked after $4 \mathrm{~h}$ and then returned almost to baseline after $16 \mathrm{~h}$, while a continuous up-regulation was observed for primary microglia in vitro (Tab. 2). A striking correlation was found for the transcripts induced by LPS in vitro and in vivo (after $4 \mathrm{~h}$ ) for some families of inflammatory mediators. For instance, there was a very high correlation for the class of interferon regulated proteins when comparing in vitro with in vivo (95\%). However, this did not hold true for all genes. In a broad analysis of several hundred genes about 33-37\% overlap has been observed over multiple gene families (Lund et al., 2006). In this context, it was a remarkable finding of the present study that the BV-2 response predicted the in vivo response with 54\% likelihood (Fig. 1B), and the genes found in vivo were also detected in BV-2 at a rate of $41 \%$. Considering the methodological limitations and the broad range of different genes, this appears to be a remarkably good overlap.

\subsection{Confirmation of mRNA expression}

Chip-based transcriptome analysis gives an overview over general regulation patterns and indicated here that many groups of genes already known from PM are also regulated in the same direction in activated BV-2. Regarding exact quantification and sensitivity, PCR is superior to the chip analysis we used. Therefore we analysed a subset of genes related particularly to host defence in the brain by quantitative PCR. Regulation of such genes similar to the pattern known from microglia would be essential for the use of BV-2 as a model for infection and inflammation in the brain. Indeed we found very high overexpression (30-200 fold) of genes such as the chemokine CCL2, interleukin-1 $\beta$, inducible nitric oxide synthase (iNOS), the inflammation marker thymidylate kinase-1 or the viral defence protein Rig-I (Fig. 2A). Also, genes for the pattern recognition receptors TLR-2, TLR-3 and NOD-2, the antioxidant enzyme glutathione reductase or the macrophage inflammation marker IFIT-3 were clearly upregulated (Fig. 2B,C), while e.g. the mRNAs for the astrocyte marker uPA or the usually constantly expressed TLR-4 were not induced. These data show that genes not detected by the broad chip approach are also regulated in $\mathrm{BV}-2$ and indicate that the overall response of these cells may actually be broader than indicated by the chip analysis. 
Tab. 2: Comparison of LPS regulated genes in BV-2 with LPS regulated genes in vivo Mice were injected i.c.v. with LPS $(2.25 \mu \mathrm{g} /$ brain) or vehicle. After 4 or $16 \mathrm{~h}$ total hippocampal RNA was purified and expression-profiled on arrays. All genes significantly regulated on the Neuroflame array are listed (historical data). The data columns indicate the ratio of up-regulation. For purposes of comparability, the table lists the data obtained in vitro from primary microglia (PM) and BV-2 cells on the same genes. Biological material was obtained from at least two independent cell- or animal experiments ( $n=6$ animals/group) and was analysed by 4 independent chip hybridisations. Neuroflame regulations were considered significant if a gene was regulated $\geq 1.8$ fold in 3 out of 4 hybridisations.

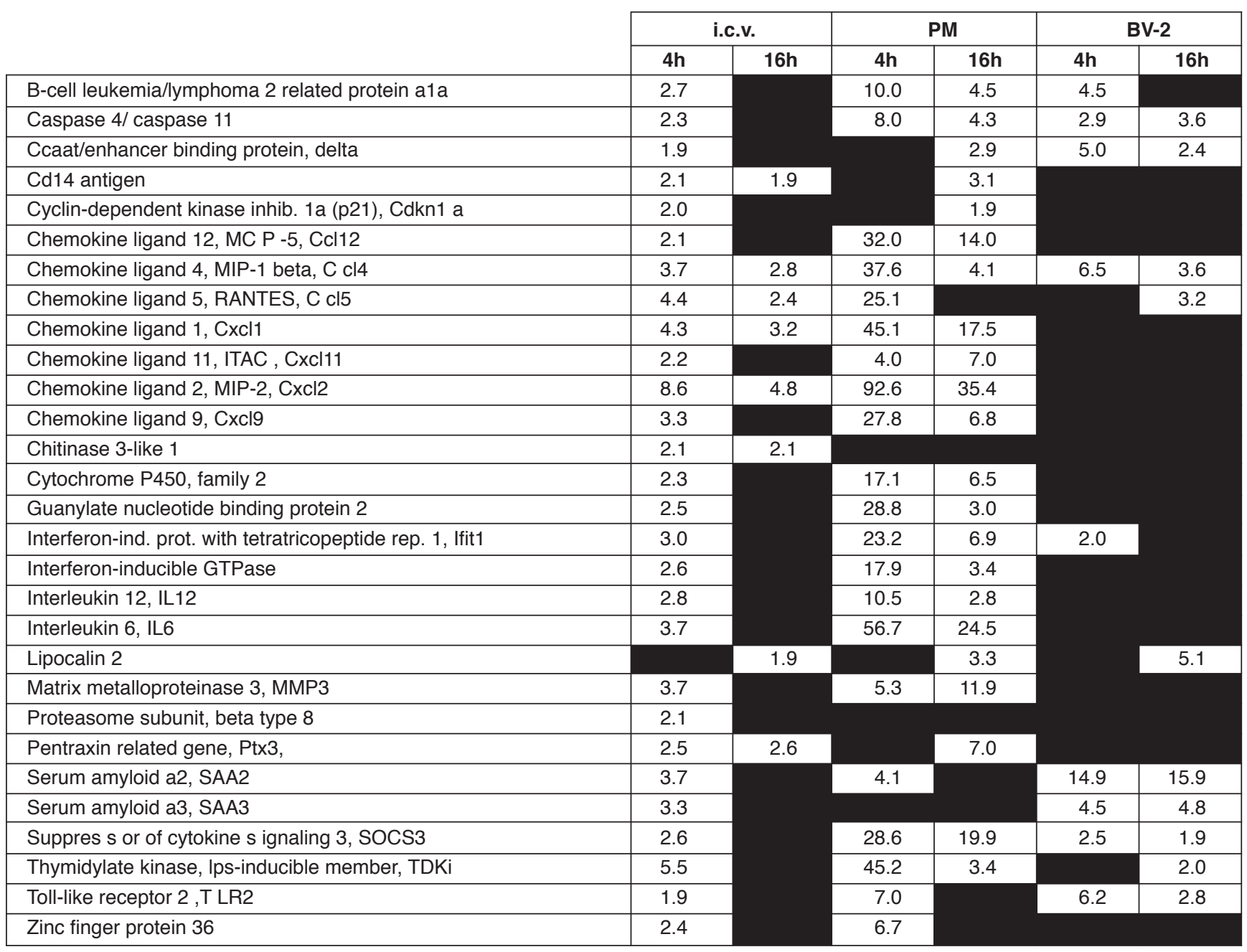

\subsection{Detection of LPS-regulated proteins in BV-2}

When inflammatory activation is examined, proteomics analysis is in many respects complementary to chip analysis. Typical inflammation markers are membrane or secreted proteins. These protein types are poorly recovered by standard (2D-gel based) proteomics approaches. On the other hand, regulation of RNAs of normal soluble proteins is often hard to detect because of their high baseline expression. Therefore, we used a proteomics approach to test whether such typical soluble markers of inflammation were detectable in activated BV-2 cells. The cells were stimulated with LPS for 24 hours and analysed by a ratiometric approach on 2D-gels. Thirty-two specifically upregulated proteins were detected. About 10 were identified by sequencing (Lund et al., 2006). For instance, manganese superoxide dismutase (SOD) (the mitochondrial inducible form of SOD; SOD-2) was clearly upregulated in LPS-stimulated BV-2. SOD-2 induction is a typical inflammation marker and was also detected on the transcriptional level in PM. In BV-2 the transcriptional changes were under the detection threshold and our proteomics findings confirm that BV-2 show a broader inflammatory response capacity than may be indicated from the chip findings with relatively hard significance rules (Fig. 3). Also, Peroxiredoxin I (Prx I) was clearly up-regulated on the protein level (Fig. 3). This protein is wellknown to be specific for glial cells (Hattori and Oikawa, 2007) and to be an indicator of microglial activation in vivo (Krapfenbauer et al., 2003; Kim et al., 2008). It was not detected by chip analysis (neither in PM nor BV-2), but shows that BV-2 indeed behave similar to microglia in vivo. 


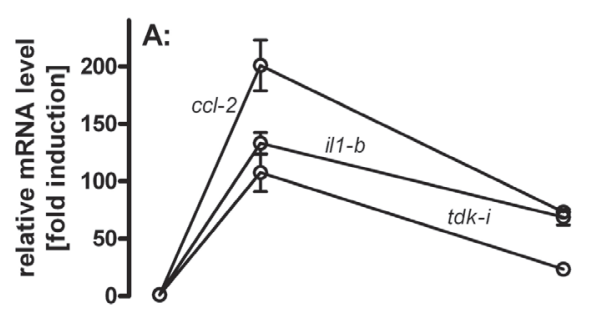

Fig. 2: Analysis of transcriptional regulation of genes associated with inflammation and host defence in BV-2 BV-2 cells were stimulated with $50 \mathrm{ng} / \mathrm{ml}$ LPS for the times indicated, before mRNA was extracted and analysed by quantitative PCR. All amplified products were first standardised to the gapdh transcript, and then the stimulation factor was calculated as relative expression of LPS treated cells and untreated cells. Data are means \pm SEM of triplicate determinations from three biological samples. Abbreviations: tdk-i = inducible thymidylate kinase, $\mathrm{ccl}-2$ = chemokine (c-c motif) ligand 2, il1-b

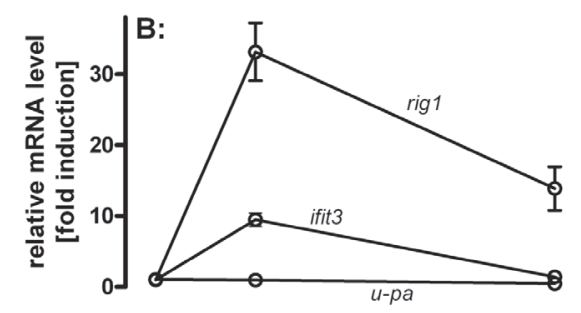
= interleukin 1 beta, rig1 = retinoic acid-inducible gene I, ifit3 = Interferon-induced protein with tetratricopeptide repeats 3, gsr1 = glutathione reductase 1, u-pa = urokinase plasminogen activator, tlr2 = toll-like receptor 2 , tlr3 = toll-like receptor 3 , tlr4 = toll-like receptor 4 , nod2 $=$ nucleotide-binding oligomerisation domain containing 2 .
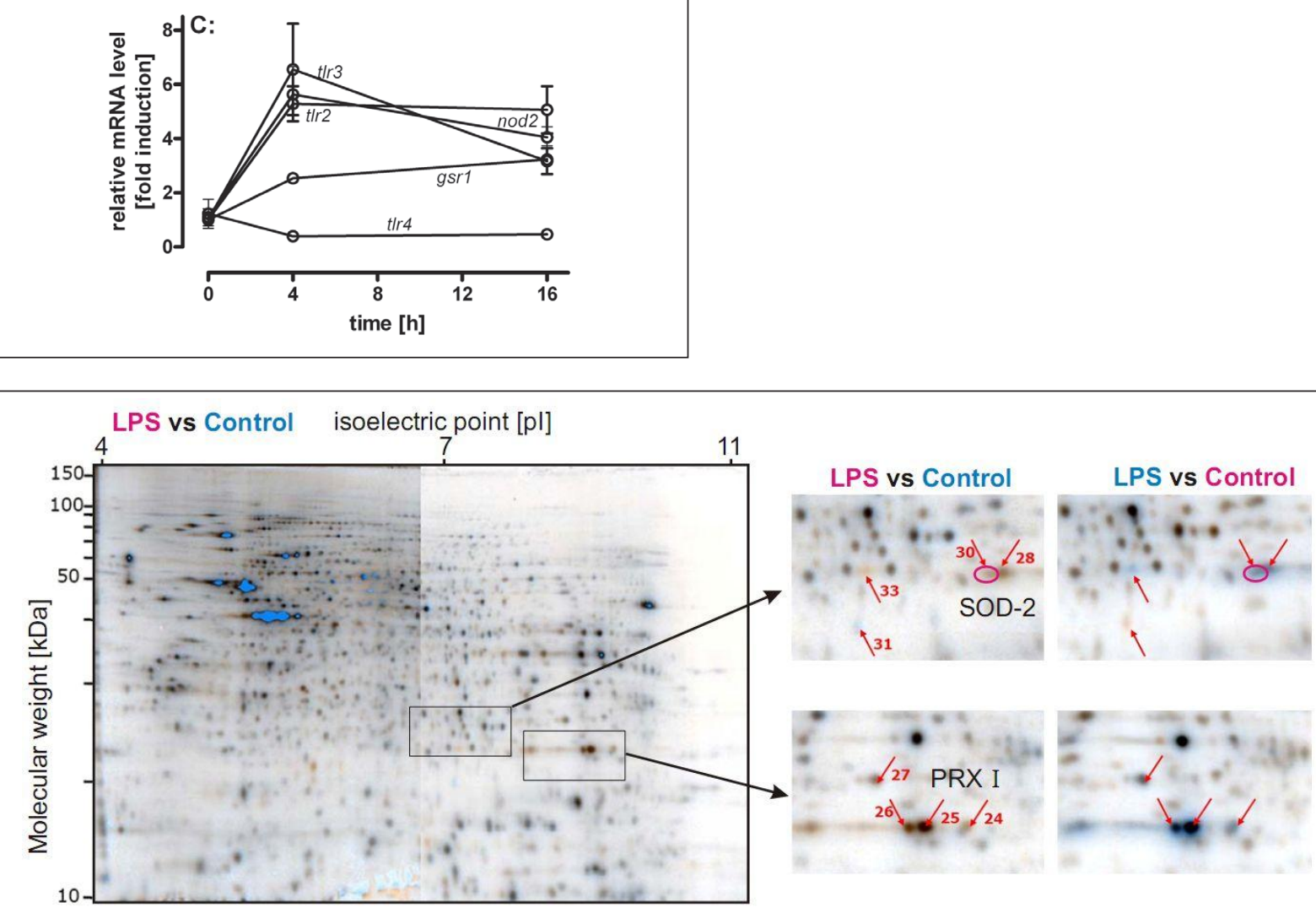

Fig. 3. Proteomics analysis of LPS-stimulated BV-2 cells

BV-2 cells were stimulated for $24 \mathrm{~h}$ with saline or LPS $(100 \mathrm{ng} / \mathrm{ml})$ before preparation of protein samples, which were subsequently separated on 2D gels. For spot quantification, samples were analysed by the proteotope method (differential labelling with iodineisotopes, remixing before the 2D run and ratio-imaging of each spot; visualised here in blue and orange). Differentially expressed proteins were identified by mass spectrometric sequencing. Total protein from four independent biological experiments was pooled and run in triplicates. One representative gel is shown (left) with blue spots being proteins more abundant in LPS-stimulated cells than in control cells. The enlargements to the right show examples of two regulated proteins with both reverse labelling approaches of the Proteotope technology. Protein spot 30 (top) was identified as the inducible form of superoxide dismutase (SOD-2). Protein spots 24-27 (bottom) are all variants of peroxiredoxin I (Prx I). Both example proteins are clearly upregulated by LPS, and this is independent of the labelling mode. Blue indicates labelling with $\mathrm{J}-125$, orange indicates labelling with $\mathrm{J}-131$. 


\subsection{Interferon responsiveness}

For the functional integration into an immune response (e.g. in the pathology of multiple sclerosis) it is important that model cells (such as BV-2) cannot only respond to inflammatory stimuli such as LPS, but also to cytokines, such as interferon gamma $(\mathrm{IFN}-\gamma)$ in an appropriate way. We examined here the responsiveness to IFN- $\gamma$ based on the known facts that induction of TNF- $\alpha$ by LPS is independent of IFN- $\gamma$ in PM, while the strong expression of iNOS, leading to high production of nitrite in the medium is strictly dependent on IFN- $\gamma$. Indeed, nitrite was only produced by BV-2 cells in the presence of the cytokine (Fig. $4 \mathrm{~A})$, and this production was very pronounced. As expected, IFN- $\gamma$ had no augmenting effect on the production of TNF- $\alpha$ after LPS stimulation (Fig. 4B). The selectivity of the induction by IFN- $\gamma$ was also analysed on the transcriptional level, where a large boost of iNOS mRNA expression was observed, while IL-1 $\beta$ was little affected, as expected from the literature on PM (Fig. 4C).

\subsection{Functional stimulation of astrocytes}

Finally, we examined the functional capacity of BV-2 in triggering cell-cell interaction. Microglia constitute the first line of defence in the brain and their acute mediators then activate astrocytes (Falsig et al., 2008). Therefore, it was essential to examine whether BV-2 were capable of activating astrocytes. We stimulated BV-2 cells with LPS and then transferred the culture supernatants $(\mathrm{CM})$ to astrocytes. These supernatants contained considerable amounts of TNF- $\alpha$, but not IL-6 (Fig. 5A). Murine astrocytes do not react to LPS (Falsig et al., 2004; Falsig et al., 2006; Falsig et al., 2008) with cytokine release or activation of the inflammation master switch NF- $\mathrm{\kappa B}$ (Fig. $5 \mathrm{~A})$. However, NF- $\mathrm{KB}$ translocation, which controls dozens of downstream inflammation events, was triggered in astrocytes by BV-2-conditioned medium (CM) (Fig. 5B). To investigate further whether NF- $\mathrm{KB}$ translocation driven by $\mathrm{CM}$ from $\mathrm{BV}-2$ indeed has functional consequences in astrocytes, we measured the release of IL-6 under the different stimulatory conditions. Again, BV-2 CM triggered a significant increase in IL-6 release (Fig. 5C). This indicates a capacity of BV-2 cells to take part in and to trigger a complex biological process usually observed under in vivo conditions.

\section{Discussion}

We examined various functions of BV-2 cells in relation to their potential role as substitute for primary microglia, and the possibility to replace in vivo experiments by cell culture models. What can be achieved by such approaches? At present, the major part of experimental animals ( $>6$ million/year in the EU) are used for basic and applied research. Thus, the stakes are very high in this area. As the biomedical research field is much less standardised than regulatory toxicology, it has been difficult to establish and validate clear 1:1 replacement systems. On the other hand, the biomedical field does not require the time-consuming and cumbersome validation process that is necessary for safety evaluations. The value of a potential replacement method in basic research is mostly determined by confidence of the scientific community in the data that can be generated. Additional factors, much more important than in the field of toxicology, are the price of the assay and the speed of data generation. Regarding the latter two parameters, BV-2 cells are clearly superior to primary microglia and animal experiments. For this reason, there are several hundred related publications, some of them involving important discoveries. These were later frequently confirmed in other cells, and would often not have been possible in vivo, or would have been technically virtually impossible with the use of PM. In many publications using BV-2 cells, the
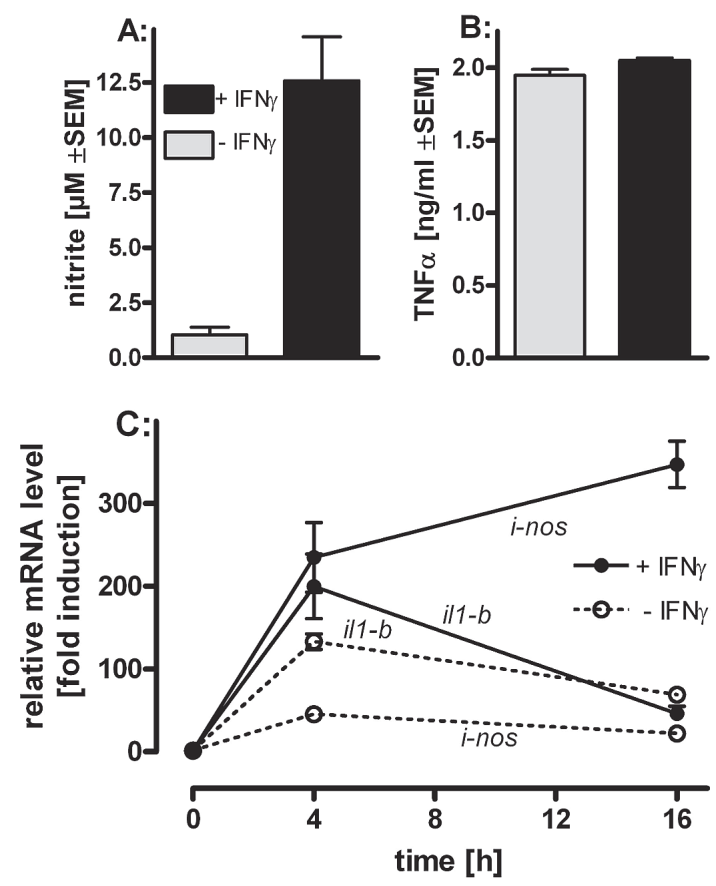

Fig. 4: Interferon-y responsiveness of BV-2 cells BV-2 cells were stimulated with $50 \mathrm{ng} / \mathrm{ml}$ LPS in the presence or absence of murine IFN- $\gamma(5 \mathrm{ng} / \mathrm{ml})$. (A) Supernatants were removed from 96 -well plates after $24 \mathrm{~h}$, and the concentration of nitrite was determined by the Griess assay. (B) Supernatants were removed from 96-well plates after $8 \mathrm{~h}$, and the concentration of TNF- $\alpha$ was determined by ELISA. (C) Cells were harvested for RNA preparation after the times indicated. Relative mRNA levels were determined by real-time PCR. Data are means \pm SEM from triplicate determinations $(n=3)$. ${ }^{*}: p \leq 0.05$ by $t$-test. inos $=$ mRNA of inducible nitric oxide synthase; il- $1 \mathrm{~b}=\mathrm{mRNA}$ of interleukin- $1 \beta$. 
data were directly compared to some data from PM to add credibility. Studies with primary microglia usually involve the use of antibiotics, at least for some time, although it is known that these can influence the outcome of experiments (Kuhlmann, 1993). We switched the BV-2 culture completely to antibioticfree conditions and also reduced the use of FBS, another source of experimental uncertainties, to $2 \%$ during stimulations. From a purely logical point-of-view, one can never be sure that a cell line will be able to replace all experiments in PM and in animals, and indeed some differences have been observed between PM and BV-2 (Hausler et al., 2002; de Jong et al., 2008). However,
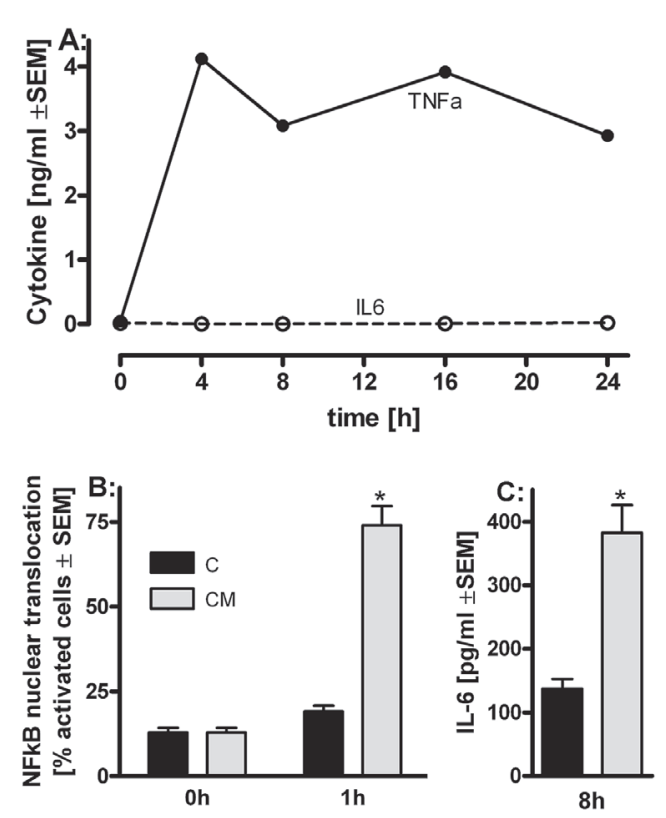

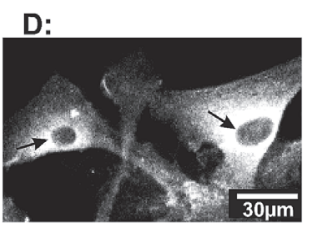

non stimulated astrocytes

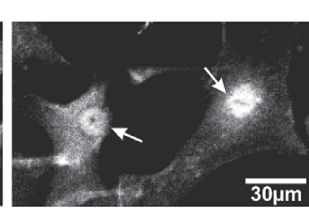

stimulated astrocytes
Fig. 5: Functional capacity of BV-2 cells to trigger astrocyte activation

BV-2 were stimulated with LPS $(50 \mathrm{ng} / \mathrm{ml})$ and the medium supernatant was removed after different incubation times. (A) $\mathrm{CM}$ was characterised at different time points for its cytokine content. TNF- $\alpha$ and IL- 6 concentrations were measured at the times indicated by ELISA. (B) Conditioned medium (CM) from the $8 \mathrm{~h}$ time point or the corresponding control medium (C, LPS added to medium after conditioning by non-stimulated BV-2 for 24 h) was added to primary astrocyte cultures. NF-кB translocation was measured after 60 minutes by image software capable of quantifying the fluorescent intensity in the nucleus and the cytosol. At least 200 cells were imaged per data point. (C) IL-6 release was measured after $8 \mathrm{~h}$. Data are means \pm SEM from triplicate determinations. *: $p \leq 0.05$ in many more cases similarities have been described. It needs to be noted that also PM have disadvantages and may yield erroneous data. It is for instance very difficult to obtain absolutely pure cultures of PM, and some contributions may arise from contaminating cells. In most cases, also the term "primary microglia" is misleading, as these cells are kept in a primary mixed glial culture for about 2 weeks after isolation, until they are re-plated and then used in secondary cultures. During the initial 2-week period, the cells phagocytose large amounts of neuronal debris left over from the isolation procedure and may thus be modified already. On top of this, it needs to be considered that the cells are usually isolated from neonatal brains and may therefore not represent adult microglia. Finally, microglia are a relatively heterogeneous cell population, with marked species and regional differences within the brain (Mahe et al., 2001; Guillemin and Brew, 2004), and thus PM may not always be representative.

This needs to be remembered when comparing the value of $\mathrm{BV}-2$ in relation to $\mathrm{PM} /$ in vivo in practical terms. The general validity of cell line models (Hartung, 2007b) vs. animal models (Hartung, 2008a) has been discussed elsewhere. Concerning the validation process (Hartung, 2007a), it is impossible to determine the value of a cell line in general, as it is impossible to compare the cell line with PM for each and every application that may have arisen in the past and may still arise in the future. An approach to this apparent dilemma is to examine the reaction pattern in certain application domains between cell line and primary cells or in vivo models. If the overall pattern is highly overlapping for a certain domain, it is likely that also in most cases not tested, a high degree of overlap would occur.

In this study we evaluated BV-2 for the use in inflammation studies. This is one of the major uses of microglia, and now doubt has been raised in this particular domain on the value of BV-2 as a model system. In a recent study, BV-2 have been compared to primary microglia, but the primary microglia were derived from rats, while BV-2 are murine cells. In many assays of this study (Horvath et al., 2008), BV-2 behaved very similar to PM, while there were mostly quantitative differences in others. The authors concluded from the relatively small data set that data from the BV-2 cell line may not be credible, if not backed by PM data (Horvath et al., 2008). In contrast to this, we observed in the past that BV-2 very often behaved similarly to PM. This was not only true for cytokine secretion, but also in very complex assays examining MAP kinase signalling (Lund et al., 2005) and phagocytosis (Hirt and Leist, 2003). Of course, occasional differences were also observed, often of quantitative nature. Therefore, we used a different type of approach for the comparison here. Instead of individual assays, we looked at the overall pattern of gene activation at the start of this study.

Basic microglial gene expression has been described for murine (Re et al., 2002) and rat primary cells (Duke et al., 2004) in addition to a murine cell line (Inoue et al., 1999). Changes in the transcriptional profile of primary rodent microglia in different conditions, such as after exposure to IFN- $\gamma$ (Moran et al., 2004), TGF- $\beta$ (Paglinawan et al., 2003) or colony-stimulating factors (Re et al., 2002), have been described. For inflammatory stimulation, data can be found for human microglia (Walker et al., 2001) or the murine BV-2 (Gan et al., 2004) cell line ex- 
posed to $\beta$-amyloid peptide and for primary microglia exposed to gram-positive bacteria (Kielian et al., 2002) or LPS (Lund et al., 2006). Data on in vivo responses are still scarce, but have been obtained for LPS (Lund et al., 2006).

The amazing finding of our study was that $90 \%$ of the genes induced in BV-2 by LPS were also found in primary microglia, and around $50 \%$ were even found in hippocampal microglia after in vivo stimulation of mice by intracerebroventricular injection of LPS. This appears to us a good overlap. However, we also observed a clear difference between PM and BV-2. PM reacted stronger to LPS and therefore a much larger (10-fold) number of genes was significantly regulated. This finding would argue for the use of BV-2 only in conditions where the correlation with PM has been confirmed by pilot data. However, we went a step further and analysed by the more sensitive PCR method a number of genes in BV-2 known to be regulated in PM. These genes were indeed regulated, although they were not detected in the chip study. Thus, there is probably less qualitative difference between PM and BV-2 than the chip study indicated. This was further confirmed by a small proteomics study, also detecting the regulation of proteins in BV-2 that are known to be regulated in PM.

The second part of the study focused on functional assays in BV-2 cells, as it has often been claimed that cell-cell interactions are especially difficult to model in vitro. Initially we looked at nitric oxide production, as this is a very frequently used parameter, and different laboratories find very different stimulation of nitric oxide synthase by LPS. This is most likely due to different co-stimulations of the cells, as inducible nitric oxide synthase is one of the two genes that has been consistently found in all stimulated microglia clones (Mahe et al., 2001). Our data show a striking difference in the $\mathrm{BV}-2$ reaction in the presence or absence of IFN- $\gamma$. This is an example of a parameter very easily controlled in BV-2 cultures, but not so easy to control in vivo or in PM, where different amounts of IFN- $\gamma$ producing cells may be involved, and thus may provide different results in different systems and laboratories. We also chose this example because it shows the limitations of using mice, and PM isolated from mice as a gold standard. BV-2 faithfully reproduce the situation known from this gold standard, but these data are not transferable to the human situation. In human microglia, nitrite production cannot be stimulated at all by LPS plus IFN- $\gamma$, and this is a fact often neglected in the hundreds of publications using murine macrophages and microglia.

In the final experiments we examined whether BV-2 are suitable for stimulation of other cells. The inflammatory response of astrocytes has been well-characterised after exposure to defined cytokines (Falsig et al., 2004; Falsig et al., 2006). In the brain microglia constitute the first line of defence (Falsig et al., 2008) and then stimulate astrocytes by a yet undefined cocktail of mediators. We show here that BV-2, stimulated by bacterial surface molecules (LPS), can indeed trigger activation in astrocytes as assessed by translocation of the transcription factor NF- $\mathrm{KB}$ and secretion of the cytokine IL-6. This experiment was possible, as our highly purified astrocytes are not reactive to LPS stimulation (Falsig et al., 2004). To our knowledge this sequence of cell interaction has not been shown in this direct form with purified cells, and the experimental system opens further fields of application of BV-2 cells.

Microglia play not only a role in inflammation, but also in classical toxicity. Especially their roles in developmental neurotoxicity have been discussed (Streit, 2001), both as modulators and as indicators of damage. This is important for current efforts to develop alternative test systems for developmental toxicity within the larger context of increasing the importance of in vitro toxicology, especially for risk assessment of environmental chemicals (Hartung and Leist, 2008; Leist et al., 2008a). The role of macrophage-like cells in organ injury and chemical toxicity is a general theme, not only for the brain, but also for other organs (Leist et al., 1998). It should be noted that BV-2 cells are already used in pharmaceutical industry (Lund et al., 2005), but the impact of alternative methods in drug discovery is still frequently underestimated (Leist et al., 2008b). Besides BV-2, also other microglia cell lines are available, although they are less frequently used. Most notable is the N9 cell line derived from the lab of Righi (Righi et al., 1989). All others are clearly less characterised. There have also been descriptions of human microglia cell lines, but none of them has undergone characterisation similar to BV-2, and their use is quite limited. To fill this gap, an alternative approach may be the generation of human microglia from stem cells, which is currently ongoing work in some laboratories and may yield interesting new in vitro systems, at least in countries where research on embryonic stem cells is possible (Leist et al., 2008c).

With all the progress made in development of improved in vitro test systems (Lehr et al., 2006; Gonzalez Hernandez and Fischer, 2007; Telang and Katdare, 2007; Thedinga et al., 2007; Wanner and Schreiner, 2008), new problems are also arising. On the one hand, systems are becoming so good and competitive that they harbour economic potential (de Brugerolle, 2007), and thus access to the cells may be limited. On the other hand, standardisation problems arise. For instance, BV-2 do not have a general problem with IL-6 production (Jang et al., 2008), but in our hands, this cytokine is hardly induced at all. This is most likely an artefact of selection and culture conditions, and demonstrates the problem of cell lines used for too long in individual laboratories. Cell lines require new stringent methods of quality control (Dirks et al., 2005), and this will be a very important issue of future research if stable alternative replacement models are to receive the confidence they need to be broadly applied also in new areas like cosmetics testing or the evaluation of food safety (Hartung, 2008b; Hartung and Koeter, 2008).

\section{References}

Baker, C. A., Martin, D. and Manuelidis, L. (2002). Microglia from Creutzfeldt-Jakob disease-infected brains are infectious and show specific mRNA activation profiles. J. Virol. 76, 10905-10913.

Blasi, E., Barluzzi, R., Bocchini, V. et al. (1990). Immortalization of murine microglial cells by a v-raf/v-myc carrying retrovirus. J. Neuroimmunol. 27, 229-237. 
Corradin, S. B., Mauel, J., Donini, S. D. et al. (1993). Inducible nitric oxide synthase activity of cloned murine microglial cells. Glia 7, 255-262.

de Brugerolle, A. (2007). SkinEthic Laboratories, a company devoted to develop and produce in vitro alternative methods to animal use. ALTEX 24, 167-171.

de Jong, E. K., de Haas, A. H., Brouwer, N. et al. (2008). Expression of CXCL4 in microglia in vitro and in vivo and its possible signaling through CXCR3. J. Neurochem. 105, 1726-1736.

Dirks, W. G., Faehnrich, S., Estella, I. A. and Drexler, H. G. (2005). Short tandem repeat DNA typing provides an international reference standard for authentication of human cell lines. ALTEX 22, 103-109.

Duke, D. C., Moran, L. B., Turkheimer, F. E. et al. (2004). Microglia in culture: what genes do they express? Dev. Neurosci. 26, 30-37.

Falsig, J., van Beek, J., Hermann, C. and Leist, M. (2008). Molecular basis for detection of invading pathogens in the brain. J. Neurosci. Res. 86, 1434-1447.

Falsig, J., Porzgen, P., Lund, S. et al. (2006). The inflammatory transcriptome of reactive murine astrocytes and implications for their innate immune function. J. Neurochem. 96, 893-907.

Falsig, J., Latta, M. and Leist, M. (2004). Defined inflammatory states in astrocyte cultures: correlation with susceptibility towards CD95-driven apoptosis. J. Neurochem. 88, 181-193.

Gan, L., Ye, S., Chu, A. et al. (2004). Identification of cathepsin $\mathrm{B}$ as a mediator of neuronal death induced by Abeta-activated microglial cells using a functional genomics approach. J. Biol. Chem. 279, 5565-5572.

Giulian, D. and Baker, T. J. (1986). Characterization of ameboid microglia isolated from developing mammalian brain. J. Neurosci. $6,2163-2178$.

Gonzalez Hernandez, Y. and Fischer, R. W. (2007). Serum-free culturing of mammalian cells - adaptation to and cryopreservation in fully defined media. ALTEX 24, 110-116.

Groebe, K., Krause, F., Kunstmann, B. et al. (2007). Differential proteomic profiling of mitochondria from Podospora anserina, rat and human reveals distinct patterns of age-related oxidative changes. Exp. Gerontol. 42, 887-898.

Guillemin, G. J. and Brew, B. J. (2004). Microglia, macrophages, perivascular macrophages, and pericytes: a review of function and identification. J. Leukoc. Biol. 75, 388-397.

Hartung, T. (2008a). Food for thought ... on animal tests. ALTEX 25, 3-16.

Hartung, T. (2008b). Food for thought ... on alternative methods for cosmetics safety testing. ALTEX 25, 147-162.

Hartung, T. (2007a). Food for thought ... on validation. ALTEX 24, 67-80.

Hartung, T. (2007b). Food for thought ... on cell culture. ALTEX 24, 143-152.

Hartung, T. and Leist, M. (2008). Food for thought ... on the evolution of toxicology and the phasing out of animal testing. ALTEX 25, 91-96.
Hartung, T. and Koeter, H. (2008). Food for thought ... on food safety testing. ALTEX 25, 259-264.

Hattori, F. and Oikawa, S. (2007). Peroxiredoxins in the central nervous system. Subcell. Biochem. 44, 357-374.

Hausler, K. G., Prinz, M., Nolte, C. et al. (2002). Interferongamma differentially modulates the release of cytokines and chemokines in lipopolysaccharide- and pneumococcal cell wall-stimulated mouse microglia and macrophages. Eur. J. Neurosci. 16, 2113-2122.

Hirt, U. A. and Leist, M. (2003). Rapid, noninflammatory and PS-dependent phagocytic clearance of necrotic cells. Cell Death Differ. 10, 1156-1164.

Horvath, R. J., Nutile-McMenemy, N., Alkaitis, M. S. and Deleo, J. A. (2008). Differential migration, LPS-induced cytokine, chemokine, and NO expression in immortalized BV-2 and HAPI cell lines and primary microglial cultures. J. Neurochem. 107, 557-569.

Inoue, H., Sawada, M., Ryo, A. et al. (1999). Serial analysis of gene expression in a microglial cell line. Glia 28, 265-271.

Jang, S., Kelley, K. W. and Johnson, R. W. (2008). Luteolin reduces IL-6 production in microglia by inhibiting JNK phosphorylation and activation of AP-1. Proc. Natl. Acad. Sci. USA 105, 7534-7539.

Kappler, J., Junghans, U., Koops, A. et al. (1997). Chondroitin/ dermatan sulphate promotes the survival of neurons from rat embryonic neocortex. Eur. J. Neurosci. 9, 306-318.

Kielian, T., Mayes, P. and Kielian, M. (2002). Characterization of microglial responses to Staphylococcus aureus: effects on cytokine, costimulatory molecule, and Toll-like receptor expression. J. Neuroimmunol. 130, 86-99.

Kim, S. U., Hwang, C. N., Sun, H. N. et al. (2008). Peroxiredoxin $\mathrm{I}$ is an indicator of microglia activation and protects against hydrogen peroxide-mediated microglial death. Biol. Pharm. Bull. 31, 820-825.

Knudsen, S. A. (2002). A biologist's guide to analysis of cDNA microarray data. New York: Wiley Interscience.

Krapfenbauer, K., Engidawork, E., Cairns, N. et al. (2003). Aberrant expression of peroxiredoxin subtypes in neurodegenerative disorders. Brain Res. 967, 152-160.

Kuhlmann, I. (1993). Zur Problematik des prophylaktischen Antibiotikaeinsatzes in Zellkultur (Problems of the prophylactic use of antibiotics in cell culture). ALTEX 10, 27-49.

Latta, M., Kunstle, G., Leist, M. and Wendel, A. (2000). Metabolic depletion of ATP by fructose inversely controls CD95and tumor necrosis factor receptor 1-mediated hepatic apoptosis. J. Exp. Med. 191, 1975-1985.

Lee, S. J. and Lee, S. (2002). Toll-like receptors and inflammation in the CNS. Curr. Drug Targets Inflamm. Allergy 1, 181-191.

Lehr, C. M., Bur, M. and Schaefer, U. F. (2006). Cell culture models of the air-blood barrier for the evaluation of aerosol medicines. ALTEX 23 Suppl., 259-264.

Leist, M., Hartung, T. and Nicotera, P. (2008a). The dawning of a new age of toxicology. ALTEX 25, 103-114.

Leist, M., Kadereit, S. and Schildknecht, S. (2008b). Food for 
thought ... on the real success of 3R approaches. ALTEX 25, 17-32.

Leist, M., Bremer, S., Brundin, P. et al. (2008c). The biological and ethical basis of the use of human embryonic stem cells for in vitro test systems or cell therapy. ALTEX 25, 163-190.

Leist, M., Gantner, F., Kunstle, G. and Wendel, A. (1998). Cytokine-mediated hepatic apoptosis. Rev. Physiol. Biochem. Pharmacol. 133, 109-155.

Leist, M., Single, B., Kunstle, G. et al. (1997). Apoptosis in the absence of poly-(ADP-ribose) polymerase. Biochem. Biophys. Res. Commun. 233, 518-522.

Lund, S., Christensen, K. V., Hedtjarn, M. et al. (2006). The dynamics of the LPS triggered inflammatory response of murine microglia under different culture and in vivo conditions. $J$. Neuroimmunol. 180, 71-87.

Lund, S., Porzgen, P., Mortensen, A. L. et al. (2005). Inhibition of microglial inflammation by the MLK inhibitor CEP-1347. J. Neurochem. 92, 1439-1451.

Mahe, D., Fisson, S., Montoni, A. et al. (2001). Identification and IFNgamma-regulation of differentially expressed $\mathrm{mR}$ NAs in murine microglial and CNS-associated macrophage subpopulations. Mol. Cell Neurosci. 18, 363-380.

Moran, L. B., Duke, D. C., Turkheimer, F. E. et al. (2004). Towards a transcriptome definition of microglial cells. Neurogenetics 5, 95-108.

Nelson, P. T., Soma, L. A. and Lavi, E. (2002). Microglia in diseases of the central nervous system. Ann. Med. 34, 491-500.

Paglinawan, R., Malipiero, U., Schlapbach, R. et al. (2003). TGFbeta directs gene expression of activated microglia to an anti-inflammatory phenotype strongly focusing on chemokine genes and cell migratory genes. Glia 44, 219-231.

Qin, H., Wilson, C. A., Lee, S. J. et al. (2005). LPS induces CD40 gene expression through the activation of NF-kappaB and STAT-1alpha in macrophages and microglia. Blood 106, 3114-3122.

Re, F., Belyanskaya, S. L., Riese, R. J. et al. (2002). Granulocyte-macrophage colony-stimulating factor induces an expression program in neonatal microglia that primes them for antigen presentation. J. Immunol. 169, 2264-2273.

Righi, M., Mori, L., De Libero, G. et al. (1989). Monokine production by microglial cell clones. Eur. J. Immunol. 19, 14431448.

Rosenberger, C. M., Scott, M. G., Gold, M. R. et al. (2000). Salmonella typhimurium infection and lipopolysaccharide stimulation induce similar changes in macrophage gene expression. J. Immunol. 164, 5894-5904.

Schrattenholz, A. and Groebe, K. (2007). What does it need to be a biomarker? Relationships between resolution, differential quantification and statistical validation of protein surrogate biomarkers. Electrophoresis 28, 1970-1979.

Streit, W. J. (2001). Microglia and macrophages in the developing CNS. Neurotoxicology 22, 619-624.
Telang, N. and Katdare, M. (2007). Cell culture model for colon cancer prevention and therapy: an alternative approach to animal experimentation. ALTEX 24, 16-21.

Thedinga, E., Ullrich, A., Drechsler, S. et al. (2007). In vitro system for the prediction of hepatotoxic effects in primary hepatocytes. ALTEX 24, 22-34.

Volbracht, C., Leist, M. and Nicotera, P. (1999). ATP controls neuronal apoptosis triggered by microtubule breakdown or potassium deprivation. Mol. Med. 5, 477-489.

Walker, D. G., Lue, L. F. and Beach, T. G. (2001). Gene expression profiling of amyloid beta peptide-stimulated human postmortem brain microglia. Neurobiol. Aging 22, 957-966.

Wanner, R. and Schreiner, M. (2008). An in vitro assay to screen for the sensitizing potential of xenobiotics. ALTEX 25, 115120 .

Weinstein, D. E. (1997). Isolation and purification of primary rodent astrocytes. In : Current Protocols in Neuroscience (unit 3.5). John Wiley and Sons Inc.

Wu, D. C., Re, D. B., Nagai, M. et al. (2006). The inflammatory NADPH oxidase enzyme modulates motor neuron degeneration in amyotrophic lateral sclerosis mice. Proc. Natl. Acad. Sci. USA 103, 12132-12137.

Yang, C. S., Lee, H. M., Lee, J. Y. et al. (2007). Reactive oxygen species and 47 phox activation are essential for the Mycobacterium tuberculosis-induced pro-inflammatory response in murine microglia. J. Neuroinflammation 4, 27.

\section{Acknowledgements}

We are indebted to many colleagues for valuable contributions and insightful discussions during the course of this work. Søren Lund, Maj Hedtjärn, Peter Pörzgen and Marcel Leist were employees of H. Lundbeck A/S (Valby, DK) during a part of this study and there used facilities and support. Anja Henn was funded by a special grant of the Land-BW. Susanne Kirner and Bettina Schimmelpfennig provided excellent technical assistance. The work was facilitated by grants from the DoerenkampZbinden Foundation and the European Community's Seventh Framework Programme (FP7/2007-2013; ESNATS project)

\section{Correspondence to}

Anja Henn

University of Konstanz

PO Box M657

78457 Konstanz

Germany

Tel.: +49-7531-885153

Fax: +49-7531-885039

e-mail: anja.henn@uni-konstanz.de 